\title{
Justicia neolinearifolia N.H. Xia \& Y.F. Deng (Acanthaceae), A NEW RECORD FOR THE FLORA OF VIETNAM
}

\author{
Do Van Hai ${ }^{1 *}$, Nguyen Khac Khoi ${ }^{1}$, Deng Yunfei ${ }^{2}$ \\ ${ }^{1}$ Institute of Ecology and Biological Resources, VAST, *dovanhaiiebr@gmail.com \\ ${ }^{2}$ South China Botanical Garden, Chinese Academy of Sciences, Guangzhou 510650, China
}

\begin{abstract}
Justicia neolinearifolia N. H. Xia \& Y.F. Deng (Acanthaceae) found in Lai Chau province is reported here for the first time for the flora of Vietnam. J. neolinearifolia can be distinguished from $J$. procumbens $\mathrm{L}$. and $J$. diffusa Willd. by having linear leaf blade and bracts. The specimens are deposited in the Herbarium of the Institute of Ecology and Biological Resources (HN) Ha Noi, Vietnam. A detailed description, illustration, important taxonomic notes, and key to the three already known species are provided.
\end{abstract}

Keywords: Acanthaceae, Justicia neolinearifolia, new record, Lai Chau, Vietnam.

\section{INTRODUCTION}

Justicia L. s.l. is one of the largest genera in Acanthaceae, which comprises about 600-800 species distributed in tropical and subtropical regions $[2,6,8]$. The genus is represented by 33 species in the Flora of Vietnam [7].

During our revisionary studies on the genus Justicia in Vietnam, we found an intersting specimen housed in the herbarium of the Institute of Ecology and Biological Resources $(\mathrm{HN})$. After critical morphological examination of the vouchers, type materials and relevant literature survey $[1,3,4,5,6,9]$, it was identified as Justicia neolinearifolia. This taxon was hitherto recorded from China, Laos and Thailand. Present work reports this as a new record for the Flora of Vietnam. A detailed description and illustration (fig.1) are provided herein.

\section{MATERIALS AND METHODS}

Specimens of Justicia housed at the following herbaria were examined: Institute of Ecology and Biological Resources, Ha Noi (HN); Institute of Tropical Biolgy, Ho Chi Minh City (VNM), VNU University of Science (HNU), South China Botanical Garden (SCBG). Our specimens of Justicia neolinearifolia were deposited in the herbarium of Institute of Ecology \& Biological Resources (HN).

\section{RESULTS AND DISCUSSION}

Key to the known allied taxa of Justicia neolinearifolia from Vietnam.

1A. Leaf blade linear (Fig. 1A); bracts linear to ca. $5 \mathrm{~mm}$ wide (Fig. 1D)

J. neolinearifolia

1B. Leaf blade elliptic, elliptic-oblong, lanceolate-elliptic, subcircular, ovate-elliptic or ovate; bracts ovate to elliptic-lanceolate, oblong-lanceolate [see Fig. 354 in Hu C. C., Deng Y. F., and Daniel T. F. (2011); Fig. 8026 in Ho, P. H. (2000)]

.2

2A. Spikes dense and cylindric, \pm densely pubescent [see Fig. 354 (1) in Hu C. C., Deng Y. F., and Daniel T. F. (2011)] J. procumbens

2B. Spikes neither dense nor cylindric, sparsely pubescent [Fig. 8026 in Ho, P. H. (2000)] ...J. diffusa

Justicia neolinearifolia N. H. Xia \& Y.F. Deng. J. Trop. Subtrop. Bot. 13(6): 534. 2005.

Type: Laos: Savannakhêt, Seno, 25 May 1952, Vidal 1730 (holotype, U, photo!) [9].
Rostellularia linearifolia Bremek. Proc. Kon. Ned. Akad. Wetensch. C 60: 5. 1957; Justicia linearifolia (Bremek.) H.S. Lo, Guihaia, 17(1): 58. 1997. non Lam. 1785; J. linearifolia subsp. liangkwangensis (H.S. Lo) H.S. Lo; Acta 
Phytotax. $\quad$ Sin. 17(4): $\quad$ 87. 1979; subsp. liangkwangensis H.S. Lo. Guihaia 17(1): J. neolinearifolia subsp. liangkwangensis (H. S. 59. 1997. Type: China. Guangxi; Nanning, 14 Lo) N. H. Xia \& Y. F. Deng, J. Trop. Subtrop. November 1935, H.Y. Liang 67184 (holotype Bot. 13(6): 534. 2005. Rostellularia linearifolia IBSC; isotype MO, photo!).

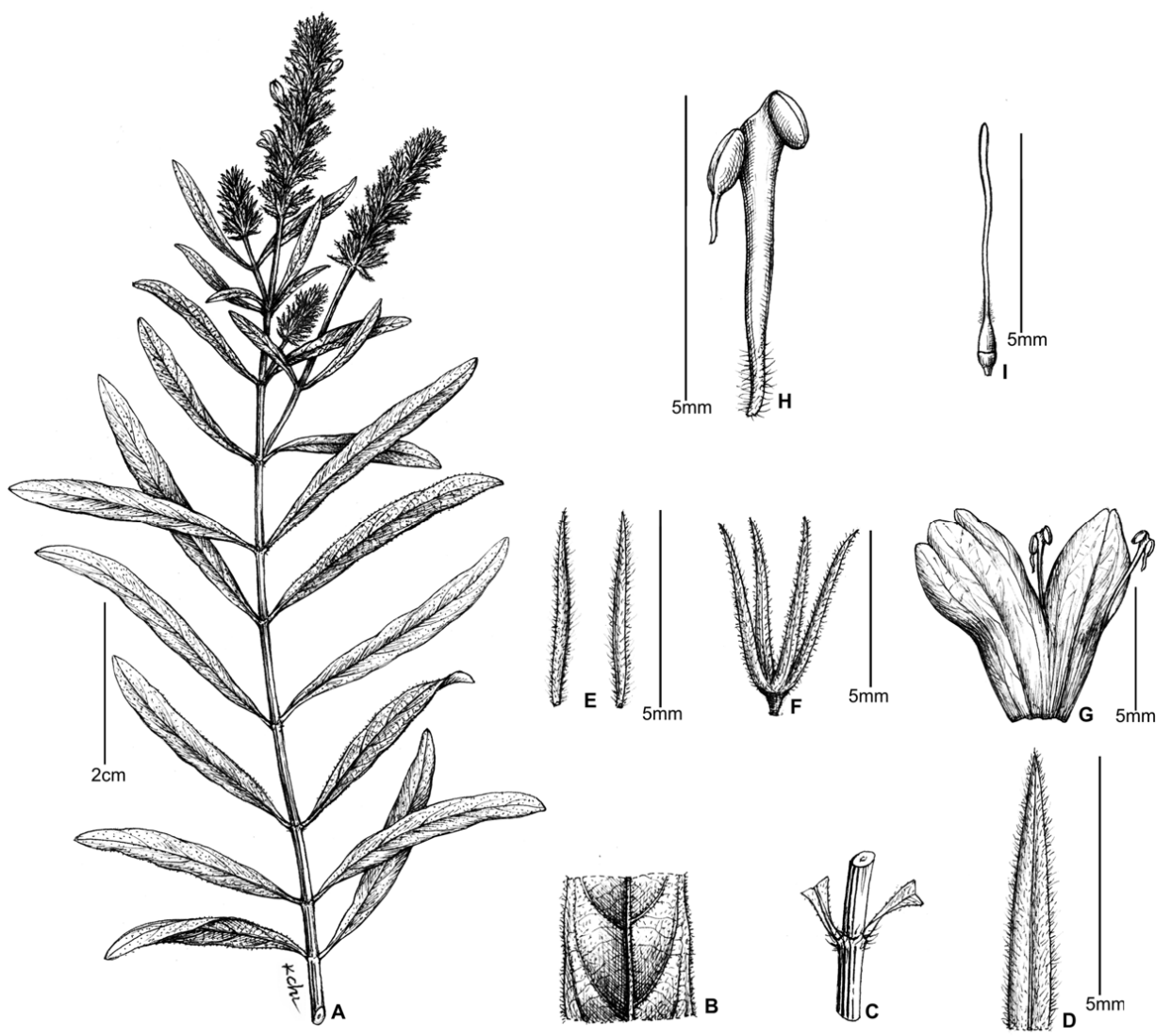

Figure 1. Justicia neolinearifolia N. H. Xia \& Y. F. Deng

A. Flowering twig; B. Leaf (a part); C. Part of stem showing petiole; D. Bract; E. Bracteole; F. Calyx; G. Corolla (open); H. Stamen; I. Ovary and style.

[Drawn by Le Kim Chi from HAL10116 (HN)]

Herbs. 30-40 cm tall, few-branched. Stems angular, sulcate, pubescent. Petiole 2-3 $\mathrm{mm}$ long, pubescent; leaf blade linear, 1.5-3.5 × 0.2$0.5 \mathrm{~cm}$, base cuneate to attenuate, margin entire, apex acute, pubescent, secondary veins 3-5 on each side of midvein. Spikes terminal, 2-3.5 cm; rachis sparsely hirsute to subglabrous. bracts linear, ca. $6 \times 1 \mathrm{~mm}$, hirsute, apex acute; bracteoles linear, ca. $5 \times 1 \mathrm{~mm}$, sparsely hirsute, apex acute, hyaline-margined, pilose along midrib, ciliate on the margin. Calyx ca. $6 \mathrm{~mm}$, outside sparsely hirsute, 4-lobed almost to base; lobes linear, apex acuminate. Corolla purplish red, ca. $8 \mathrm{~mm}$, 2-lipped, lower lip 3-lobed, upper lip 2-lobed. Stamens 2; filaments ca. 5 $\mathrm{mm}$, sparsely hirsute toward the base; anther thecae superposed, lower one spurred at base. Ovary hirsute; style ca. $5 \mathrm{~mm}$, hirsute at base. Capsule ca. $6 \mathrm{~mm}$, pubescent, solidly stalked on the basal portion. Seeds ovate, ca. $1 \times 1 \mathrm{~mm}$, surface verrucose. Fl. and Fr. October December.

Distribution: Vietnam: Lai Chau: Muong Te. China, Thailand, Laos. 
Ecology and habitat: The plant was found growing in secondary evergreen forest on very steep rocky slopes and cliffs at elevations of 200-300 m.a.s.1.

Additional specimens examined: VIETNAM. Lai Chau Province: Muong Te district, Muong Te Municipality, Nam Cum village, 24 November 2006 Nguyen Tien Hiep, L.Averyanov, Pham Van The, HAL10116 (HN); Muong Te district, 25 November 2006 Nguyen Tien Hiep, L. Averyanov, Pham Van The, HAL10135 (HN).

Note: Justicia neolinearifolia belongs to sect. Rostellaria which is characterized by the terminal spikes and 4-partitate calyx. In vietnam, two speices belonging to this sectionviz. $J$. procumbens L. and $J$. diffusa Wild. have hitherto been reported. $J$. neolinearifolia can be differentiated from them by having linear leaf blade and bracts.

Present report will help prioritizing conservation of this species in Vietnam.

\section{REFERENCES}

1. Fang D., Lo X. R., Tang H. T., 1997: New materials for Acanthaceae from Guangxi. Guihaia, 17(1): 23-59 (in Chinese).
2. Graham V. A. W., 1988: Delimitation and infra-generic classification of Justicia (Acanthaceae). Kew Bulletin 43: 551-624.

3. Guangdong Institute of Botany, 1975. Flora Hainanica, Tome 3: 1-629. Beijing: Science Press (in Chinese).

4. Phạm Hoàng Hộ, 2000: Cây cỏ Việt Nam, Quyển 3: 75-81. Nxb. Trẻ, tp. Hồ Chí Minh.

5. Hu C. C., 2002: Florae Reipublicae Popularis Sinicae, Tomus, 70: 1-309. Beijing: Science Press (in Chinese).

6. Hu C. C., Deng Y. F., Daniel T. F., 2011: Justicia L. In: Flora of China, Vol. 19: 449461. Science Press, Beijing and Missouri Botanical Garden Press, St. Louis.

7. Trần Kim Liên, 2005: Acanthaceae: Danh lục các loài thực vật Việt Nam, 3: 251-281. Nxb. Nông nghiệp, Hà Nội.

8. Mabberley D. J., 2008: Mabberley's plantbook: A portable dictionary of plants, their classification and uses. 3rd edition, 1021 pp. Cambridge University Press, New York.

9. Xia N. H., Deng Y. F., 2005: Nomecalatural Novelties in Justicia Linn. (Acanthaceae) from China, J. Trop. Subtrop. Bot., 13(6): 533-534.

\title{
GHI NHẬN MỚI LOÀI Justicia neolinearifolia N.H. Xia \& Y.F. Deng CHO HỆ THỬC VẬT VIẸT NAM
}

\author{
Đỗ Văn Hài ${ }^{1}$, Nguyễn Khắc Khôi ${ }^{1}$, Deng Yunfei ${ }^{2}$ \\ ${ }^{1}$ Viện Sinh thái và Tài nguyên sinh vật, Viện Hàn lâm KH\&CN Việt Nam \\ ${ }^{2}$ Vườn Thực vật Hoa Nam, Viện Hàn lâm Khoa học Trung Quốc
}

\section{TÓM TẮT}

Chi Xuân tiết (Justicia L.) là một trong những chi lớn với nhiều loài, gồm khoảng 600-800, loài phân bố ở vùng nhiệt đới và cận nhiệt đới. Ở Việt Nam, đến nay đã biết 33 loài [8]. Trong quá trình nghiên cứu chi Xuân tiết ở Việt Nam, chúng tôi phát hiện loài Justicia neolinearifolia N.H. Xia \& Y.F. Deng, bổ sung cho hệ thực vật Việt Nam. Mẫu vật nghiên cứu được thu thập ở tỉnh Lai Châu và lưu giữ tại phòng tiêu bản thực vật (HN), Viện Sinh thái và Tài nguyên sinh vật. 
Loài Justicia neolinearifolia thuộc nhánh Rostellaria với đặc điểm cụm hoa bông ở đầu cành hoặc nách lá; đài 4 thùy. Trong nhánh này ở Việt Nam hiện có 3 loài và đây là loài ghi nhận mới. Loài J. neolinearifolia có đặc điểm khác biệt với các loài khác bởi có lá bắc và phiến lá hình đường. Bài báo cũng đưa ra khóa định loại cho 3 loài này thuộc chi Justicia có ở Việt Nam.

Tù khóa: Acanthaceae, Justicia neolinearifolia, ghi nhận mới, Việt Nam.

Ngày nhận bài: 15-7-2015 